\title{
Physical exercise promotes proliferation and differentiation of endogenous neural stem cells via ERK in rats with cerebral infarction
}

\author{
WEI LIU, WEN WU, GUANGYONG LIN, JIAN CHENG, YANYAN ZENG and YU SHI \\ Department of Rehabilitation Medicine, Zhujiang Hospital, Southern Medical University, \\ Guangzhou, Guangdong 510280, P.R. China
}

Received November 22, 2017; Accepted May 14, 2018

DOI: $10.3892 / \mathrm{mmr} .2018 .9147$

\begin{abstract}
Physical exercise is beneficial for the functional recovery of neurons after stroke. It has been suggested that exercise regulates proliferation and differentiation of endogenous neural stem cells (NSCs); however, the underlying molecular mechanisms are still largely unknown. In the present study, the aim was to investigate whether physical exercise activates the extracellular signal-regulated kinase (ERK) signaling pathway to promote proliferation and differentiation of NSCs in rats with cerebral infarction, thereby improving neurological function. Following middle cerebral artery occlusion, rats underwent physical exercise and neurological behavior was analyzed at various time points. Immunofluorescence staining was performed to detect proliferation and differentiation of NSCs, and western blotting was used to analyze cyclin-dependent kinase 4 (CDK4), Cyclin D1, retinoblastoma protein (p-Rb), P-16, phosphorylated (p)-ERK1/2 and c-Fos expression. The results indicated that physical exercise promoted proliferation and differentiation of NSCs, and led to improved neural function. In addition, the expression levels of CDK4, Cyclin D1, p-Rb, p-ERK1/2 and c-Fos were upregulated, whereas the expression of P-16 was downregulated following exercise. U0126, an inhibitor of ERK signaling, reversed the beneficial effects of exercise. Therefore, it may be hypothesized that physical exercise enhances proliferation and differentiation of endogenous NSCs in the hippocampus of rats with cerebral infarction via the ERK signaling pathway.
\end{abstract}

Correspondence to: Professor Wen Wu, Department of Rehabilitation Medicine, Zhujiang Hospital, Southern Medical University, 253 Industrial Avenue, Guangzhou, Guangdong 510280, P.R. China

E-mail: wuwen66@163.com

Key words: physical exercise, endogenous NSCs, ERK, MCAO, U0126

\section{Introduction}

Functional recovery after cerebral infarction is a complex phenomenon that is dependent on brain plasticity. Studies have demonstrated that endogenous neural stem cells (NSCs) proliferate, migrate and differentiate following cerebral infarction, and are involved in regeneration of nervous tissue and recovery of brain function (1-3). NSCs have been the focus of much research; however, the neurobiological mechanisms that control proliferation and differentiation of endogenous NSCs after cerebral ischemia remain unclear.

Physical exercise is widely used as a rehabilitation treatment for promoting sensory and motor function recovery in patients after stroke. The recovery mechanisms are generally believed to be associated with neural plasticity (4). In rats, physical exercise has been demonstrated to reduce infarct volume, promote angiogenesis and induce neurogenesis $(5,6)$; in addition, Luo et al (7) reported that physical exercise can promote proliferation of NSCs or precursor cells in rat brain tissue. Most studies to date have only observed the effect of physical exercise on NSCs in the hippocampus following cerebral infarction; therefore, further studies are required to better understand the underlying molecular mechanisms.

The role of various signal transduction pathways in the mechanism of cerebral ischemic injury has garnered much attention. Notably, activation of the extracellular signal-regulated kinase (ERK) pathway appears to be implicated in ischemic brain injury (8). Ischemia, hypoxia, growth factors and other factors can lead to activation of ERK, which in turn translocates to the nucleus, and increases the expression of genes associated with cell proliferation and differentiation $(9,10)$. It has previously been identified that pre-exercise training can upregulate the ERK signaling pathway and improve neurological function (11); however, it is unknown whether physical exercise following cerebral ischemia can still activate the ERK signaling pathway. At present, there are different views on the timing and mechanism of physical exercise, and physical exercise following cerebral infarction is more in line with clinical practice. Therefore, the present study investigated the effects of physical exercise $24 \mathrm{~h}$ after cerebral infarction in rats. The aim was to investigate whether physical exercise could promote proliferation and 
differentiation of NSCs in the dentate gyrus of rats with cerebral infarction, and improve neurological function by activating the ERK signaling pathway.

Exploring the role of physical exercise and ERK signaling in endogenous NSCs will aid in determining the molecular mechanisms associated with physical exercise, and may explain the specific regulatory mechanism of the ERK signaling pathway. The results may also provide novel targets for clinical rehabilitation, further promote the application of physical exercise in the clinic and provide a more solid theoretical foundation of kinesiotherapy.

\section{Materials and methods}

Animals. Normal adult male Sprague Dawley rats (250-300 g, 3-4 months old) were provided by the Experimental Animal Center of Southern Medical University (Guangzhou, China). The rats were given enough water and food every day at $22^{\circ} \mathrm{C}$, 3-5 rats per cage. Procedures involving animals and their care were conducted in accordance with National Institute of Health (NIH) guidelines (NIH pub. no. 85-23, revised 1996), and the present study was approved by the Animal Care and Use Committee of the Southern Medical University (Guangzhou, China).

Middle cerebral artery occlusion (MCAO) model. Rat cerebral ischemia reperfusion injury was performed according to the Longa method of MCAO (12). Rats were anesthetized by intraperitoneal injection of $10 \%$ chloral hydrate $(400 \mathrm{mg} / \mathrm{kg})$. The skin on the neck was shaved, sterilized and a $2 \mathrm{~cm}$ midline incision was made. The right carotid artery, external carotid artery and internal carotid artery were identified and carefully separated. Subsequently, the external carotid artery and common carotid artery near the heart were ligated. Briefly, suture thread was inserted through the carotid artery into the internal carotid artery for a distance of $19 \pm 0.5 \mathrm{~mm}$ until resistance was felt, to occlude the origin of the MCA. Following $2 \mathrm{~h}$, the suture was withdrawn to allow reperfusion. In the sham surgery group, rats were processed in the same way as the MCAO group, however vessels were not ligated and no occlusion suture was inserted. Following completion of the operation, rats were placed in clean housing for recovery. All procedures were performed under aseptic conditions.

Verification of the MCAO model. To verify the reliability and reproducibility of the MCAO model using the Longa method, triphenyl tetrazolium chloride (TTC) staining was performed to detect brain lesions in two rats that were randomly selected from the MCAO group. Firstly, rats were anesthetized with $10 \%$ chloral hydrate $(500 \mathrm{mg} / \mathrm{kg})$ and the heart was then exposed. An infusion needle was inserted through the left ventricle to cannulate the aorta and cut the right atrium, the blood was flushed by perfusion with $\sim 500 \mathrm{ml}$ saline. Once the liquid was clear, the rats were decapitated; the brain was dissected, placed in the refrigerator of $-20^{\circ} \mathrm{C}$ for quick freezing 20-30 min and placed in a customized slicer to cut $2 \mathrm{~mm}$ coronal sections. Sections were incubated in $2 \%$ TTC solution at $37^{\circ} \mathrm{C}$ in the dark for $30 \mathrm{~min}$ and then removed. The stained sections were photographed immediately.
Inclusion criteria and experimental groups. Rat neurological findings following MCAO were evaluated using the Longa scoring method (12): 0 , no neurological deficit; 1 , failure to extend left forepaw fully indicating mild neurological deficit; 2 , circling to the left indicating moderate focal neurological deficit; 3 , falling to the left indicating severe focal deficit; and 4, no spontaneous walking and depressed level of consciousness. Animals with scores of 1-3 were included in the present study.

Rats were randomly divided into the physical exercise group ( $\mathrm{E} ; \mathrm{n}=27)$, the physical exercise group receiving U0126, a mitogen-activated protein kinase kinase (MEK) 1/2 inhibitor that blocks ERK signaling (EU; $n=27)$, the control group (MCAO but untreated) $(\mathrm{C}, \mathrm{n}=27)$, the control group treated with $\mathrm{U} 0126$ (CU; $\mathrm{n}=27)$, and the sham surgery group $(\mathrm{S} ; \mathrm{n}=27)$. These five groups were further divided into three subgroups with time points of 7, 14 and 21 days after MCAO.

Physical exercise. The E and EU groups underwent treadmill adaptation exercise training on an electric treadmill (length, $45 \mathrm{~cm}$ ), 2 days prior to the MCAO surgery. Rats in each group were housed in standard cages after surgery ( $n=3-5$ rats/cage). Rats in the C, CU and S group were fed ad libitum, and allowed to move freely in the cage. Rats in the E and EU group began exercise training $24 \mathrm{~h}$ after MCAO surgery. The treadmill was not inclined and was set to a speed of $10-20 \mathrm{~m} / \mathrm{min}$; and the rats ran $30 \mathrm{~min} /$ day five times a week. Animals were sacrificed at the respective time points for tissue analysis.

Neurological severity scores. Modified neurological severity score (mNSS) tests were used to assess the neurological function of the rats in each group at 7,14 and 21 days after MCAO surgery (13). There were six items in the mNSS test: Spontaneous activity test, a paresis test, a forelimb motor function test, a motor function test, and tests for pain sensation and deep sensation, with a total score of 18. The neurological function was graded on a scale of 0-18: 13-18 indicated severe injury, 7-12 indicated moderate injury and 1-6 indicated mild injury. The tests were performed blind and in triplicate, and the average score was recorded.

\section{5-bromodeoxyuridine (BrdU) administration and tissue collec-} tion. A total of 3 days prior to tissue collection, rats in each group were intraperitoneally injected with $\mathrm{BrdU}(50 \mathrm{mg} / \mathrm{kg}$; Sigma-Aldrich; Merck KGaA, Darmstadt, Germany) three times a day at $8 \mathrm{~h}$ intervals (for 2 days). Animals were sacrificed $24 \mathrm{~h}$ after the last injection. U0126 (Cell Signaling Technology, Inc., Danvers, MA, USA) was dissolved in dimethyl sulfoxide, diluted to $0.5 \mathrm{mg} / \mathrm{ml}$ in $\mathrm{PBS}$, and injected into the tail vein of $\mathrm{CU}$ and EU rats $(0.5 \mathrm{mg} / \mathrm{kg}) 30 \mathrm{~min}$ prior to MCAO surgery.

Rats were anesthetized with $500 \mathrm{mg} / \mathrm{kg} 10 \%$ chloral hydrate via intraperitoneal injection. The chest was opened to expose the heart, and the brain was fixed by cardiac perfusion with $4 \%$ paraformaldehyde in PBS for $24 \mathrm{~h}$ at $4^{\circ} \mathrm{C}$. Brains were dissected following decapitation, and the right hemispheres were paraffin-embedded. Paraffin blocks were serially sliced in the coronal plane into 5- $\mu \mathrm{m}$ sections; every fifth section was used for staining.

Hematoxylin and eosin $(H \& E)$ staining. H\&E staining was used to detect MCAO-induced lesions at the respective time 
points. Deparaffinization of tissue sections was achieved by conventional xylene dewaxing, ethanol removal of xylene and washing in distilled water for $2 \mathrm{~min}$. The sections were placed in hematoxylin for $1 \mathrm{~min}$, washed in water for $1 \mathrm{~min}$, differentiated in $1 \%$ hydrochloric acid ethanol for 10-30 sec, washed in water for $20 \mathrm{~min}$, placed in eosin for 5-10 min all at room temperature and then dehydrated using an ethanol gradient. The sections were fixed with neutral balata for 12-24 h at room temperature and visualized under a light microscope.

Immunofluorescence staining. Immunofluorescence staining was used to detect $\mathrm{BrdU}^{+} /$neuronal nuclei $(\mathrm{NeuN})^{+}$and $\mathrm{BrdU}^{+} / \mathrm{glial}$ fibrillary acidic protein $(\mathrm{GFAP})^{+}$cells in the hippocampal dentate gyrus at 7, 14 and 21 days after MCAO surgery. Deparaffinized sections were incubated in $3 \% \mathrm{H}_{2} \mathrm{O}_{2}$ in methanol solution for $10 \mathrm{~min}$ and then trypsin-digested for $10 \mathrm{~min}$ at $37^{\circ} \mathrm{C}$. Subsequently, the sections were microwaved in citrate buffer ( $\mathrm{pH}$ 6.0) for antigen retrieval and blocked with normal goat serum (Boster Biotechnology, Inc., Wuhan, China) for $10 \mathrm{~min}$ at $37^{\circ} \mathrm{C}$. The sections were incubated at $4^{\circ} \mathrm{C}$ overnight with the following primary antibodies: Mouse anti-BrdU (1:100; cat. no. B2531; Sigma-Aldrich; Merck KGaA), rabbit anti-NeuN (1:200; cat. no. ab177487) and rabbit anti-GFAP (1:500; cat. no. ab7260; both Abcam, Cambridge, MA, USA). Following a wash step in PBS, the sections were incubated with secondary antibodies for $1 \mathrm{~h}$ at $37^{\circ} \mathrm{C}$ : Cy3 goat anti-mouse $\operatorname{IgG}$ (1:300; cat. no. TA130012; OriGene Technologies, Inc., Beijing, China) and Alexa Fluor 488 goat anti-rabbit IgG (1:400; cat. no. ab150077; Abcam). Lastly, DAPI (cat. no. D9542; Sigma-Aldrich; Merck $\mathrm{KGaA}$ ) was added for $10 \mathrm{~min}$ at room temperature, sections were washed in PBS and mounted in 50\% glycerol. Negative controls were generated by replacing the primary antibodies with PBS. Sections were examined using an Olympus inverted microscope, and five non-overlapping visual fields were selected at random. Images were analyzed using Image-Pro Plus version 6.0 (Media Cybernetics, Inc., Rockville, MD, USA), and the number of $\mathrm{BrdU}^{+} / \mathrm{NeuN}^{+}$and $\mathrm{BrdU}^{+} / \mathrm{GFAP}^{+}$cells $/ \mathrm{mm}^{2}$ of hippocampal dentate gyrus slice was counted.

Western blot analysis. Western blotting was used to detect cyclin-dependent kinase 4 (CDK4), Cyclin D1, retinoblastoma protein (p-Rb), P-16, phosphorylated (p)-ERK1/2 and c-Fos protein expression in the hippocampus 7,14 and 21 days after MCAO surgery. To extract proteins, the hippocampus was incubated in RIPA lysis buffer (Beyotime Institute of Biotechnology, Haimen, China). The sample was centrifuged and the supernatant was harvested for protein quantification using bicinchoninic acid assay. A total of $50 \mu \mathrm{g}$ proteins were separated by $12 \%$ polyacrylamide gel electrophoresis and transferred onto a polyvinylidende difluoride membrane. $5 \%$ skimmed milk powder was incubated with the membrane at room temperature for $2 \mathrm{~h}$. Membranes were incubated at $4^{\circ} \mathrm{C}$ overnight with antibodies against: CDK4 (1:1,000; cat. no. ab199728; Abcam), Cyclin D1 (1:1,000; cat. no. 2978), p-Rb (1:1,000; cat. no. 8516; both Cell Signaling Technology, Inc.), P-16 (1:1,000; cat. no. ab54210; Abcam), ERK (1:1,000; cat. no. 4695), p-ERK (1:2,000; cat. no. 4370; both Cell Signaling Technology, Inc.), c-Fos (1:4,000; cat. no. ab134122; Abcam) or $\beta$-actin (1:1,000; cat. no. 4970; Cell Signaling Technology, Inc.). Subsequently, the membranes were washed and incubated with HRP-conjugated goat anti-rabbit IgG antibody $(1: 2,500$; cat. no. TA140003; OriGene Technologies, Inc. Beijing, China) for $1 \mathrm{~h}$ at $37^{\circ} \mathrm{C}$. Proteins were visualized and analyzed by the Bio-Rad ChemiDoc ${ }^{\mathrm{TM}}$ XRS+ gel imaging system (Bio-Rad Laboratories, Inc., Hercules, CA, USA) and the software is ImageJ v1.8 (National Institutes of Health, Bethesda, MD, USA). Protein expression was normalized against the internal reference protein, $\beta$-actin.

Statistical analysis. Statistical analyses were conducted using SPSS version 20.0 software (IBM Corp., Armonk, NY, USA). All values were expressed as the means \pm standard deviation. The experiments were repeated three times. One-way analysis of variance was used to determine whether there was a statistically significant difference between multiple groups. The Levene's test was used to assess the equality of variances. If the variance was homogeneous, the Fisher's least significant difference test was used and if the variance was not homogeneous the Dunnett's test was used. $\mathrm{P}<0.05$ was considered to indicate a statistically significant difference.

\section{Results}

Preparation of the MCAO model. TTC staining of the rat brains confirmed that the MCAO was successfully established. As shown in Fig. 1A, MCAO generated infarction in the brain, which was indicated by a pale white color. Conversely, normal brain tissue was pink in color, indicating living tissue.

Functional assessment. The mNSS scores were zero for the $\mathrm{S}$ group at all time points. The mNSS score for the E group was significantly lower compared with in the $\mathrm{C}$ and $\mathrm{CU}$ groups 7 days following MCAO surgery $(\mathrm{P}<0.05)$, and although the score was also lower than in the EU group, significance was not reached. At 14 and 21 days after MCAO, the E group had a significantly lower mNSS score compared with all other experimental groups $(\mathrm{P}<0.05)$. The $\mathrm{mNSS}$ score of the $\mathrm{C}$ group was significantly lower compared with in the CU group 14 and 21 days after MCAO $(\mathrm{P}<0.05$; Fig. $1 \mathrm{~B})$.

Pathological alterations. H\&E staining (Fig. 2) revealed that the cells in the cerebral cortex and hippocampus of the $\mathrm{S}$ group were large and neatly arranged, with distinct nuclear membranes and nucleoli. In addition, the nucleus was located in the center of the cells, and the tissue was intact.

In the $\mathrm{C}, \mathrm{CU}$ and EU groups, the right cerebral cortex and hippocampal area contained ischemic lesions, tissue edema, cell degeneration and necrosis. The normal morphology of some neurons was lost. The cytoplasm, nuclear membrane and nucleoli appeared abnormal, with deeply stained nuclei and nuclear condensation.

Pathological alterations were alleviated in the E group. The nerve cells were generally neatly arranged, with normal cell size and morphology. The cytoplasm was stained blue and the nucleoli were clear.

Expression of $\mathrm{BrdU}^{+} / \mathrm{NeuN}^{+}$cells in the hippocampal dentate gyrus. The majority of cells in the $\mathrm{S}$ group were $\mathrm{BrdU} / \mathrm{NeuN}^{+}$, indicating that proliferation was minimal; only a small number of $\mathrm{BrdU}^{+} / \mathrm{NeuN}^{+}$cells were observed in some brain 
A

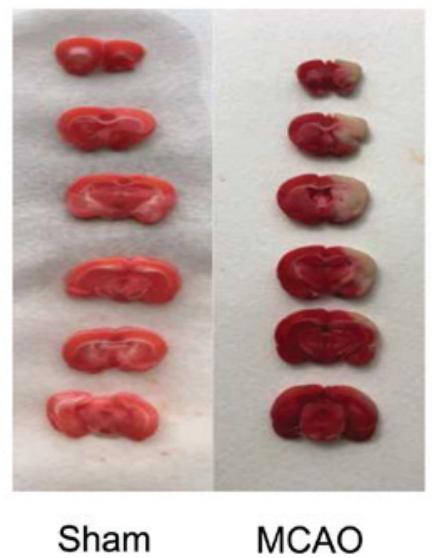

B

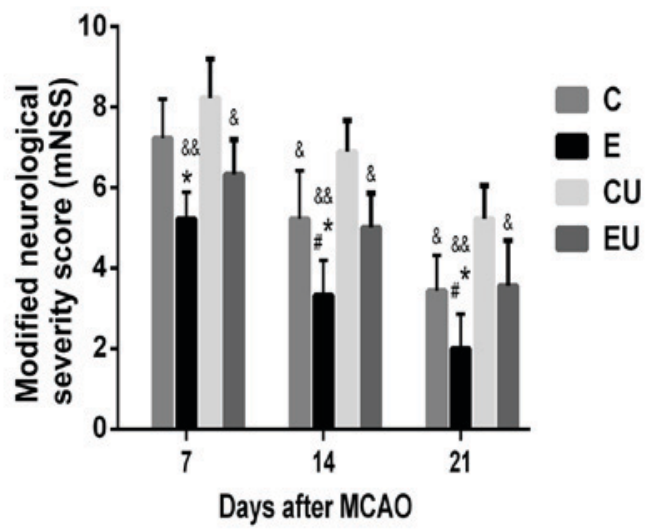

Figure 1. mNSS following MCAO. (A) Representative coronal sections of triphenyltetrazolium chloride staining of brain tissue isolated from the sham and MCAO groups, $24 \mathrm{~h}$ following surgery. (B) Neurological function was evaluated by mNSS 7, 14 and 21 days after MCAO. Data are presented as the means \pm standard deviation. ${ }^{*} \mathrm{P}<0.05$ vs. $\mathrm{C} ;{ }^{*} \mathrm{P}<0.05$ vs. EU; ${ }^{\star} \mathrm{P}<0.05$, ${ }^{\&}{ }^{\mathrm{P}} \mathrm{P}<0.001$ vs. CU. C, control group; CU, control group treated with U0126; E, physical exercise group; EU, physical exercise group treated with U0126; MCAO, middle cerebral artery occlusion; mNSS, modified neurologic severity score.

S

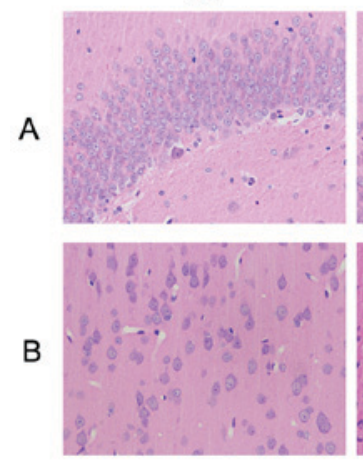

C
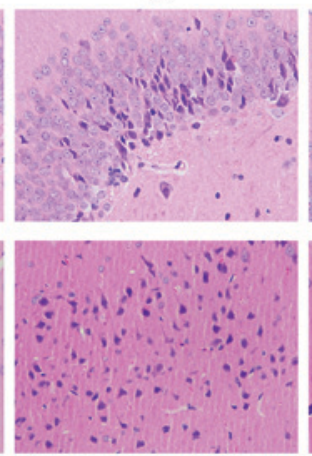

$E$

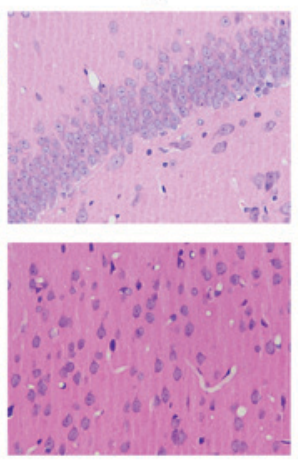

CU
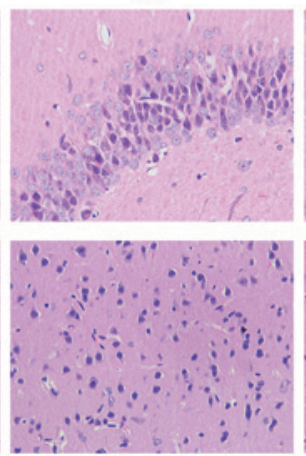

EU

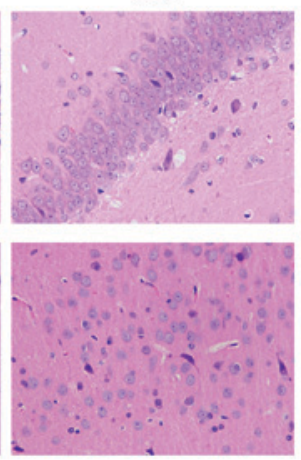

Figure 2. Hematoxylin and eosin staining of the (A) hippocampal dentate gyrus and (B) cerebral cortex, 7 days after MCAO. C, control group; CU, control group treated with U0126; E, physical exercise group; EU, physical exercise group treated with U0126; MCAO, middle cerebral artery occlusion; S, sham surgery group.

slices. The number of $\mathrm{BrdU}^{+} / \mathrm{NeuN}^{+}$cells in the $\mathrm{E}$ group was significantly higher compared with in the $\mathrm{C}$ and $\mathrm{CU}$ groups 7 days after MCAO $(\mathrm{P}<0.05)$; and the number of cells was also higher compared with the EU group, however there was no statistically significant difference. The E group demonstrated a significant increase in the number of $\mathrm{BrdU}^{+} / \mathrm{NeuN}^{+}$cells compared with all other groups 14 and 21 days after MCAO surgery $(\mathrm{P}<0.05)$. The number of cells in the $\mathrm{C}$ group was significantly higher than the $\mathrm{CU}$ group at 14 and 21 days after MCAO (P<0.05; Fig. 3).

Expression of $\mathrm{BrdU}^{+} / \mathrm{GFAP}^{+}$cells in the hippocampal dentate gyrus. Most cells in the $\mathrm{S}$ group were $\mathrm{BrdU}^{+} / \mathrm{GFAP}^{+}$with only a small number of $\mathrm{BrdU}^{+} / \mathrm{GFAP}^{+}$cells observed in some brain slices. The E group had a significant increase in the number of $\mathrm{BrdU}^{+} / \mathrm{GFAP}^{+}$cells 7,14 , and 21 days after $\mathrm{MCAO}(\mathrm{P}<0.05)$, indicating proliferating astrocytes. The number of cells in the $\mathrm{C}$ group was significantly higher compared with in the $\mathrm{CU}$ group 14 and 21 days after MCAO ( $\mathrm{P}<0.05$; Fig. 4$)$.

Western blot analysis. The expression levels of Cyclin D1 in the E group were significantly increased at all time points compared with in the other groups $(\mathrm{P}<0.05)$. The expression levels of CDK4 and $\mathrm{p}-\mathrm{Rb}$ in the $\mathrm{E}$ group were significantly increased 14 and 21 days compared with in the other groups $(\mathrm{P}<0.05)$. Although the expression levels of CDK4 and $\mathrm{p}-\mathrm{Rb}$ in the $\mathrm{E}$ group were increased at 7 days compared with in the $\mathrm{C}$ group, the difference was not statistically significant. The expression levels of Cyclin D1, CDK4 and p-Rb in the $\mathrm{C}$ group were significantly higher compared with the $\mathrm{CU}$ group 7, 14, and 21 days after MCAO $(\mathrm{P}<0.05)$. The expression levels of P-16 in the E group were significantly lower at 14 days compared with in the other groups $(\mathrm{P}<0.05)$, and the expression levels of P-16 in the E group were lower at 7 and 21 days compared with in the $\mathrm{C}$ group, but the difference was not statistically significant. The expression levels of P-16 in the $\mathrm{C}$ group were significantly lower than in the $\mathrm{CU}$ group 7 and 14 days after MCAO ( $\mathrm{P}<0.05$; Fig. 5).

The expression levels of p-ERK1/2 and c-Fos in the E group were significantly increased at each time point compared with in the other groups $(\mathrm{P}<0.05)$. The expression levels of $\mathrm{c}-\mathrm{Fos}$ in the $\mathrm{C}$ group were significantly higher compared with in the $\mathrm{CU}$ group 7, 14, and 21 days after $\mathrm{MCAO}(\mathrm{P}<0.05)$. The expression levels of $\mathrm{p}$-ERK1/2 in the $\mathrm{C}$ group were significantly higher compared with in the $\mathrm{CU}$ group 7 and 21 days after MCAO ( $\mathrm{P}<0.05$; Fig. 6). 
A
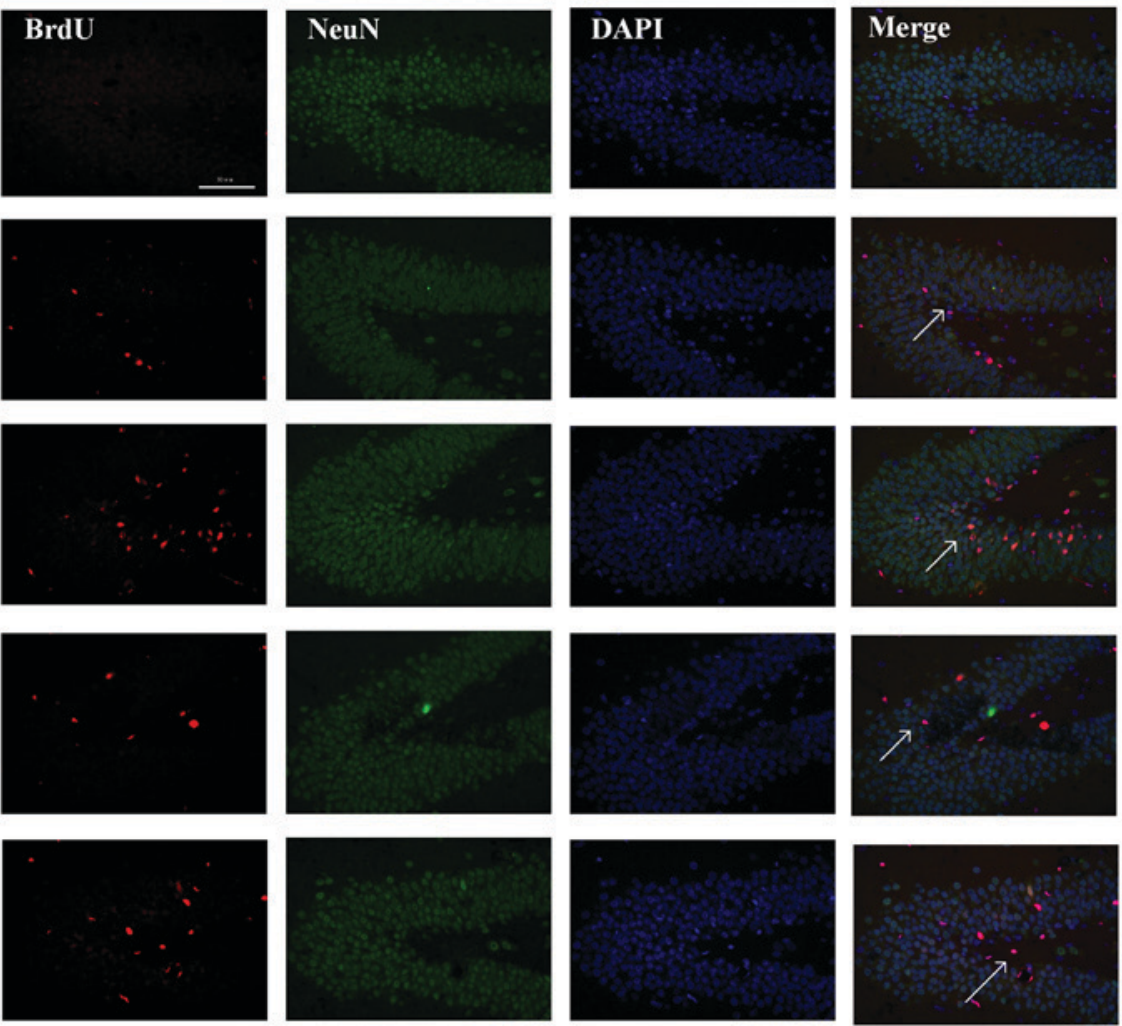

CU

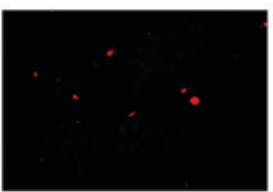

EU
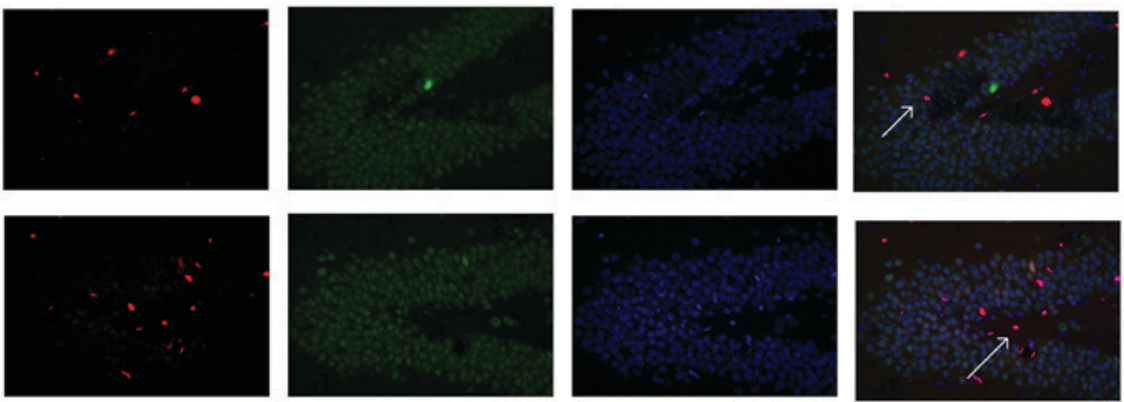

B

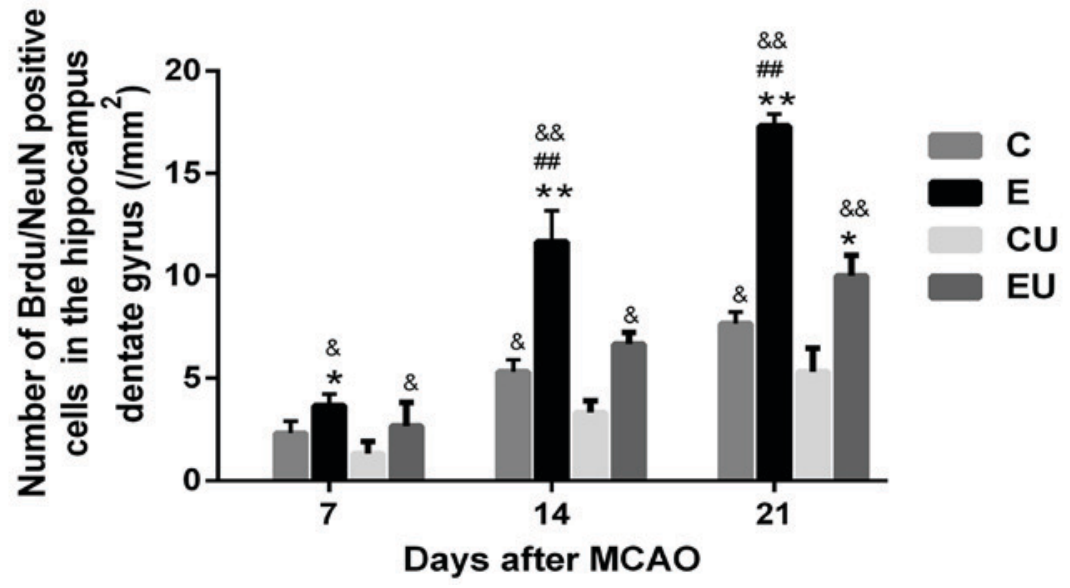

Figure 3. Exercise enhances proliferation of neurons in the hippocampal dentate gyrus. (A) Immunofluorescence staining for BrdU (red) and NeuN (green) in rats 21 days after MCAO. Scale bar $=50 \mu \mathrm{m}$. (B) Quantification of BrdU $\mathrm{NeuN}^{+}$cells 7, 14 and 21 days after $\mathrm{MCAO}$. Data are presented as the means \pm standard deviation. The arrows in the images are indicating $\mathrm{BrdU}^{+} / \mathrm{NeuN}^{+}$cells. ${ }^{*} \mathrm{P}<0.05,{ }^{* *} \mathrm{P}<0.001$ vs. C; ${ }^{\# \prime} \mathrm{P}<0.001$ vs. EU; ${ }^{\&} \mathrm{P}<0.05$, \&\& $\mathrm{P}<0.001$ vs. CU. BrdU, bromodeoxyuridine; C, control group; CU, control group treated with U0126; E, physical exercise group; EU, physical exercise group treated with U0126; MCAO, middle cerebral artery occlusion; NeuN, neuronal nuclei.

\section{Discussion}

Studies have demonstrated that physical exercise serves an important role in recovery of neurological function in patients with cerebral infarction (14-16). The rehabilitation mechanism of physical exercise is widely believed to be associated with neural plasticity, and may be related to the regulation of differentiation and proliferation of endogenous NSCs; however, the molecular mechanisms still need to be clarified.

BrdU is a thymine nucleotide analog that is incorporated into DNA during the $\mathrm{S}$ phase of the cell cycle and is a commonly used marker for cellular proliferation (17). Zhang et al (18) demonstrated that this is a common method for detecting NSCs. Previous studies have demonstrated that the presence of increased numbers of $\mathrm{BrdU}^{+}$cells identified the proliferation of NSCs $(19,20)$. In the present study, proliferating cells were visualized by immunofluorescence double staining. $\mathrm{BrdU}^{+} / \mathrm{NeuN}^{+}$and $\mathrm{BrdU}^{+} / \mathrm{GFAP}^{+}$cells identified proliferating neurons and proliferating astrocytes, respectively. Due to the presence of increased numbers of $\mathrm{BrdU}^{+} / \mathrm{NeuN}^{+}$and $\mathrm{BrdU}^{+} / \mathrm{GFAP}^{+}$cells, and decreased mNSS scores in the $\mathrm{E}$ group, it may be hypothesized that physical exercise enhances the proliferation and differentiation of endogenous NSCs to 
A
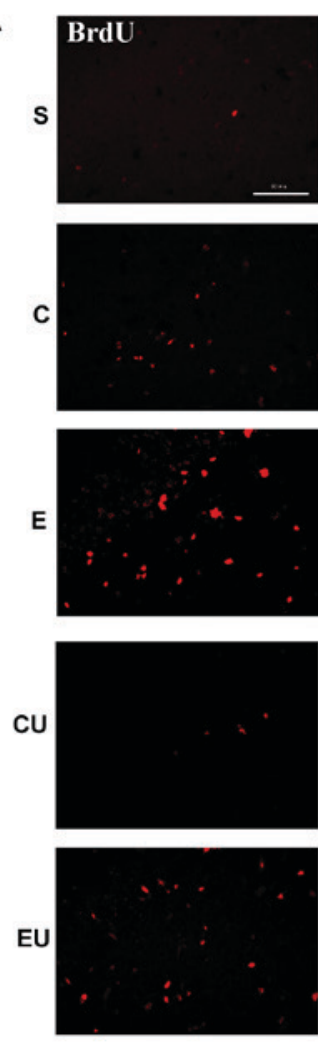
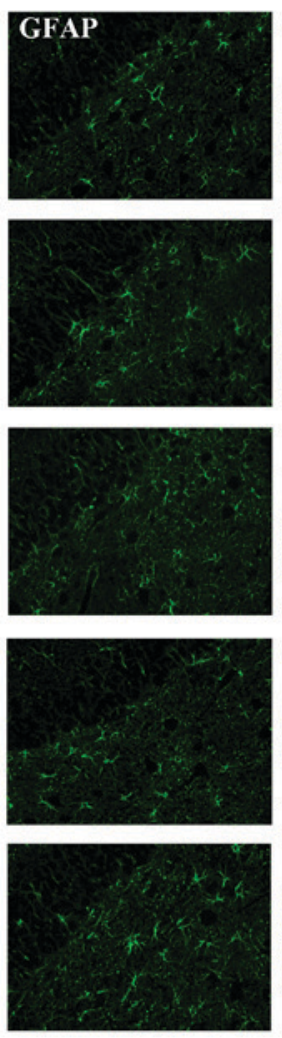
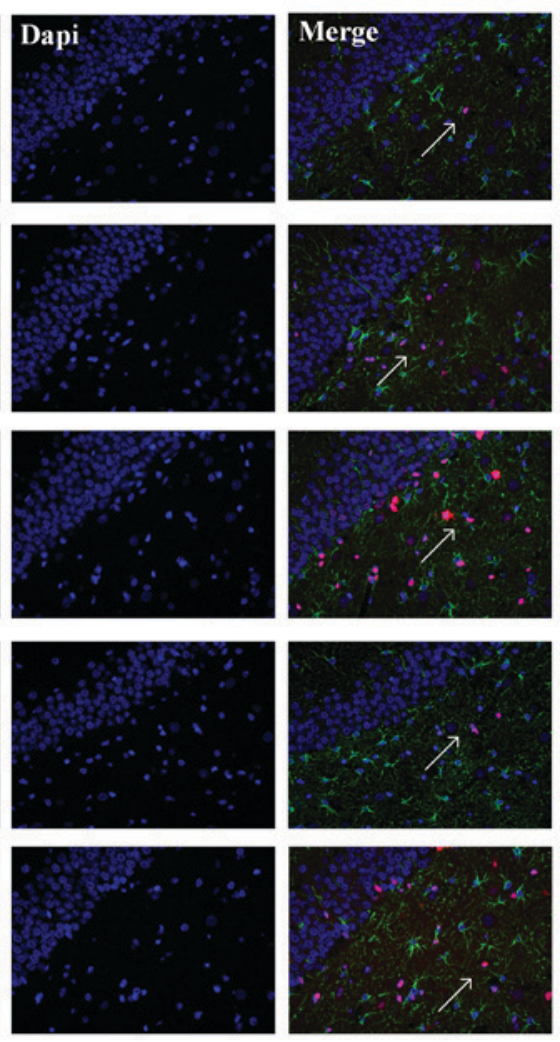

B

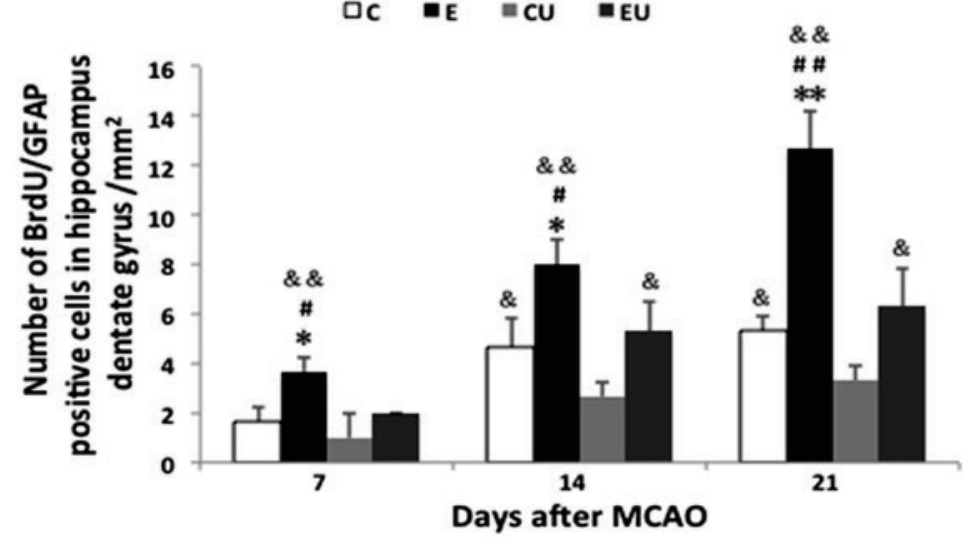

Figure 4. Exercise enhances proliferation of astrocytes in the hippocampal dentate gyrus. (A) Immunofluorescence staining for BrdU (red) and GFAP (green) in rats 21 days after MCAO. Scale bar $=50 \mu \mathrm{m}$. (B) Quantification of $\mathrm{BrdU}^{+} / \mathrm{GFAP}^{+}$cells 7, 14 and 21 days after MCAO. The arrows in the images are indicating $\mathrm{BrdU}^{+} / \mathrm{GFAP}^{+}$cells. Data are presented as the means \pm standard deviation. ${ }^{*} \mathrm{P}<0.05,{ }^{* *} \mathrm{P}<0.001$ vs. $\mathrm{C} ;{ }^{\#} \mathrm{P}<0.05,{ }^{\# \#} \mathrm{P}<0.001$ vs. EU; ${ }^{\&} \mathrm{P}<0.05$, ${ }^{\& \&} \mathrm{P}<0.001$ vs. CU. BrdU, bromodeoxyuridine; C, control group; CU, control group treated with U0126; E, physical exercise group; EU, physical exercise group treated with U0126; GFAP, glial fibrillary acidic protein; MCAO, middle cerebral artery occlusion.

improve the neural function of rats with cerebral infarction. Injection of U0126, an ERK signaling pathway inhibitor, suppressed the proliferation of neural cells and affected the mNSS score. The experimental results demonstrated that physical exercise not only promoted differentiation of endogenous NSCs into neurons, but also their differentiation into astrocytes. It is thought that astrocytes develop a scar around the ischemic region, thereby hindering the growth of new neurons; however, growing evidence suggests that activated astrocytes can inactivate excitatory glutamate and secrete nerve growth factor (NGF), which supports neural cell survival and axon growth (21). Only live astrocytes can support extension of axonal growth cones, thus potentially compensating for injured nerve cells $(22,23)$. According to the mNSS results, astrocytes may have a role in endogenous neuroprotection and neural recovery after cerebral ischemia, but the mechanisms need to be further studied.

Clinical and animal studies have revealed that physical exercise can promote nerve regeneration (24-27). Ferreira et al (28) demonstrated that short-term and moderate treadmill exercise in rats with cerebral infarction improves hippocampal plasticity, and Brandt et al (29) identified that physical exercise promotes the proliferation of NSCs and maturation of neurons in the dentate gyrus of rats, which are in line with the results obtained in the present study. BrdU labeling of proliferation is affected by dose, frequency and other factors. In our previous experiment, BrdU was injected 1 day prior to animal sacrifice (30), marking fewer proliferative cells, 
A

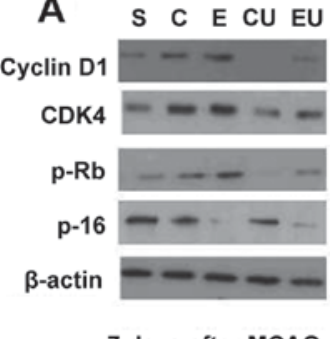

7 days after MCAO
B S C E CU EU

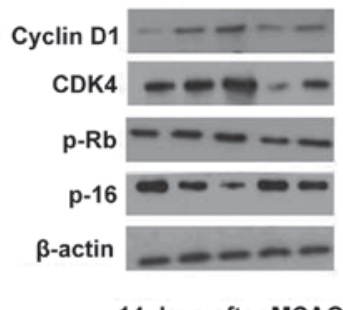

C S C E CU EU

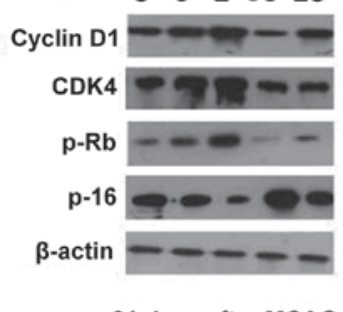

21 days after MCAO
D

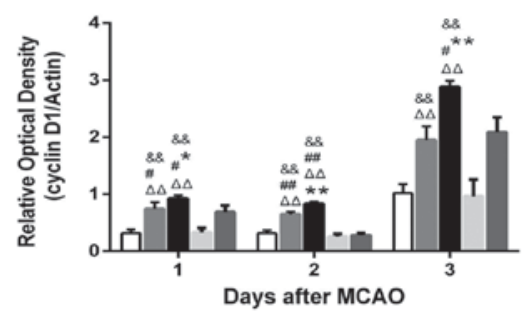

F

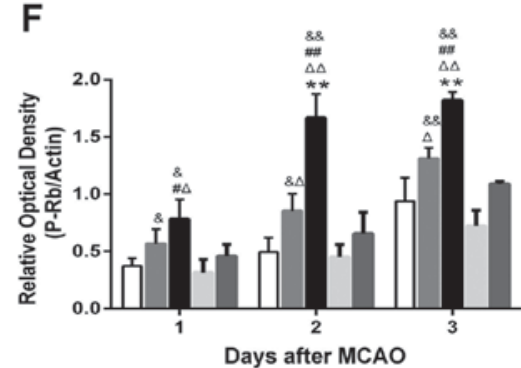

E

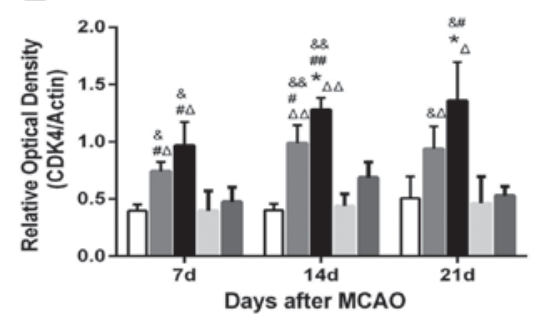

G

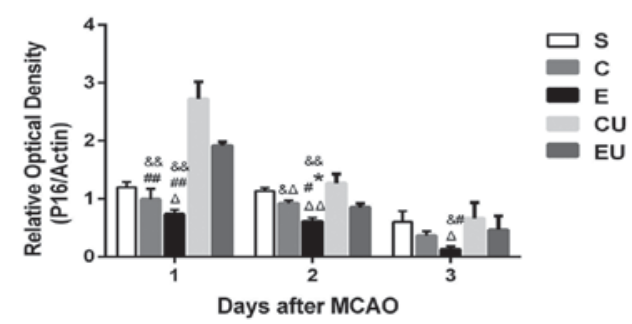

Figure 5. Western blot analysis of Cyclin D1, CDK4, p-Rb and P-16 in the hippocampal tissue of rats (A) 7, (B) 14, (C) 21 days after MCAO. Relative expression of (D) Cyclin D1, (E) CDK4, (F) p-Rb and (G) P-16 7, 14 and 21 days after MCAO. Data are presented as the means \pm standard deviation. ${ }^{*} \mathrm{P}<0.05$, ${ }^{* *} \mathrm{P}<0.001$ vs. $\mathrm{C} ;{ }^{\#} \mathrm{P}<0.05$, ${ }^{\# \#} \mathrm{P}<0.001$ vs. EU; ${ }^{\&} \mathrm{P}<0.05$, ${ }^{\&} \mathrm{P}<0.001$ vs. $\mathrm{CU} ;{ }^{\Delta} \mathrm{P}<0.05,{ }^{\Delta \Delta} \mathrm{P}<0.001$ vs. S. C, control group; CDK4, cyclin-dependent kinase 4 ; CU, control group treated with U0126; E, physical exercise group; EU, physical exercise group treated with U0126; MCAO, middle cerebral artery occlusion; p-Rb, retinoblastoma protein; $\mathrm{S}$, sham surgery group.

whereas daily injection of BrdU may exert potential toxicity in differentiating stem cells (7). Therefore, in the present study, BrdU was injected 3 days prior to animal sacrifice, to mark cell proliferation and avoid toxicity; however, whether this injection scheme is the best remains to be determined.

The proliferation of endogenous NSCs is defined by cells entering the next phase of the cell cycle from the resting $\mathrm{G}_{0}$ phase. Cell cycle proteins, including cyclins and CDKs, can control the progression of cells through the cell cycle by forming a complex, thereby initiating activation. Conversely, CDK inhibitors (CKIs) suppress CDK activity and act as negative regulators (31). For example, Cyclin D1 and CDK4 can form a complex known as Cyclin D1/CDK4, which phosphorylates substrate protein $\mathrm{p}-\mathrm{Rb}$ to drive cells through the cell cycle $\mathrm{G}_{1} / \mathrm{S}$ checkpoint. The CKI, P-16, binds to CDK4 and blocks its interaction with Cyclin D1, resulting in inhibition of $\mathrm{p}-\mathrm{Rb}$ phosphorylation, as well as inhibition of cell proliferation (32). The results presented in this study revealed that physical exercise upregulated the expression of CDK4, Cyclin D1 and p-Rb, but downregulated the expression of P-16. The use of U0126 inhibited the induction of cell proliferation-associated proteins by physical exercise. Therefore, it may be hypothesized that physical exercise promotes progression of NSCs from the $\mathrm{G}_{0} / \mathrm{G}_{1}$ to $\mathrm{S}$ phase by regulating cell proliferation-related protein expression leading to proliferation, with the ERK signaling pathway potentially having an important regulatory role.

ERK is a member of the mitogen-activated protein kinase (MAPK) family. Hypoxia, ischemia, inflammatory cytokines, growth factors, mechanical stress, and biochemical and physical factors can activate the ERK signaling pathway, resulting in a series of phosphorylation processes. The tyrosine kinase receptor Raf- 1 binds to Ras and activates the latter, which in turn phosphorylates its downstream substrate MEK. Phosphorylation of MEK activates the downstream phosphorylation of ERK1/2. p-ERK is the active form of ERK that can not only phosphorylate cytosolic proteins, but also transcription factors, including c-Fos, c-Jun, Elk-1 and c-Myc (33). These transcription factors participate in various processes, including cell differentiation, proliferation, inflammation and oxidative stress (34). In cerebral ischemia, the expression and activation of ERK is caused by growth factors, $\mathrm{Ca}^{2+}$ influx, oxygen free radicals and excitatory amino acids. ERK is downstream of the NGF receptor and through sequential activation of NGF receptor-Ras-Raf-MEK promotes nerve regeneration (35). Studies have demonstrated that activation of the ERK pathway inhibits neuronal excitotoxicity and serves a neuroprotective role, whereas blocking activation of the ERK pathway promotes cell death (36). Poddar and Paul (37) also 
A

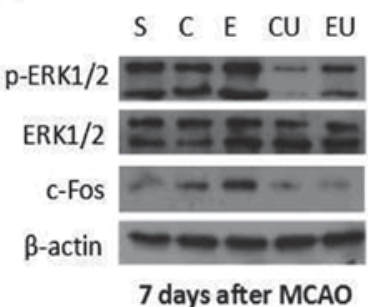

B

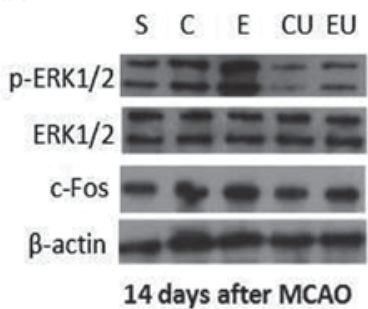

C

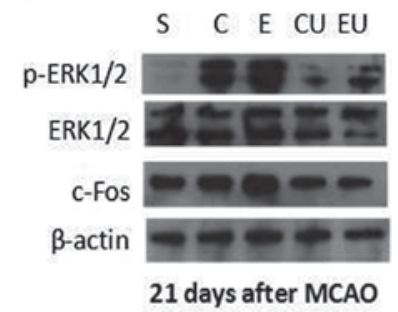

D

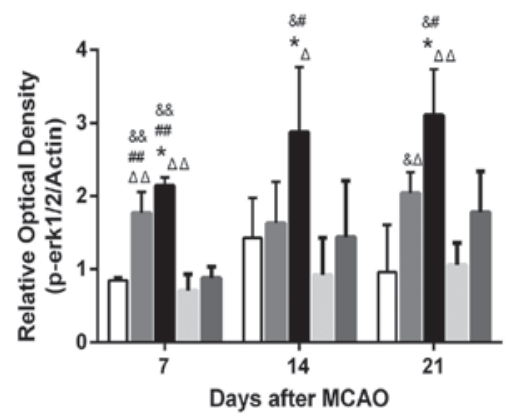

E

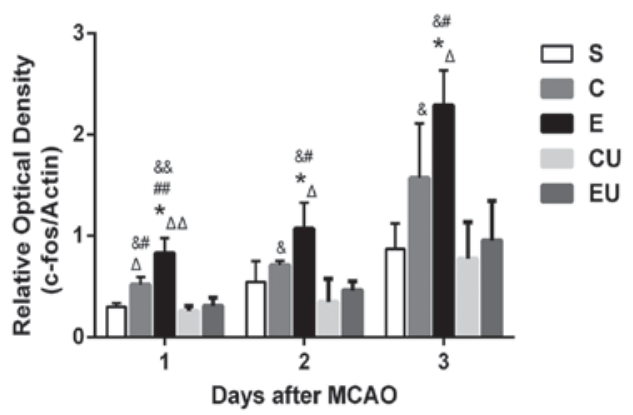

Figure 6. Western blot analysis of p-ERK1/2, ERK1/2 and c-Fos in the hippocampal tissue of rats (A) 7, (B) 14 and (C) 21 days after MCAO. Relative protein expression of (D) p-ERK1/2 and (E) c-Fos 7, 14 and 21 days after MCAO. Data are presented as the means \pm standard deviation. ${ }^{*} \mathrm{P}<0.05$ vs. $\mathrm{C}$; ${ }^{*} \mathrm{P}<0.05$, ${ }^{\# \#} \mathrm{P}<0.001$ vs. EU; ${ }^{\circledR} \mathrm{P}<0.05,{ }^{\&} \& \mathrm{P}<0.001$ vs. $\mathrm{CU} ;{ }^{\Delta} \mathrm{P}<0.05,{ }^{\Delta \Delta} \mathrm{P}<0.001$ vs. S. C, control group; CU, control group treated with U0126; E, physical exercise group; ERK, extracellular signal-regulated kinase; EU, physical exercise group treated with U0126; MCAO, middle cerebral artery occlusion; p, phosphorylated; $\mathrm{S}$, sham surgery group.

observed that inhibition of ERK phosphorylation reduces neuronal cell death. Therefore, ERK activation appears to have an important dual role in brain protection and injury.

In rats with spinal cord injury, it has been established that physical exercise activates the ERK signaling pathway leading to regeneration of axons and improved motor function (38). Studies have also demonstrated that electric treadmill training can improve neurological function in rats after cerebral ischemia, which may be related to activation of the ERK signaling pathway; however, the activation pathway remains largely unknown (39). In the present study, it was identified that the protein expression levels of p-ERK1/2 and c-Fos in the E group were higher compared with in the other experimental groups 7, 14 and 21 days after MCAO. When the MEK1/2 inhibitor U0126 was applied, the expression levels of p-ERK1/2 and the downstream protein c-Fos were diminished. These results indicated that physical exercise upregulated the ERK signaling pathway and U0126 blocked the phosphorylation of ERK1/2 caused by physical exercise. This suggested that physical exercise has a positive regulatory role in promoting the proliferation and differentiation of endogenous NSCs via the ERK signaling pathway. Notably, Li et al (11) demonstrated that pre-exercise training in mice may upregulate the exercise-induced hormone irisin, which activates the ERK signaling pathway and improves neurological function. The present study identified the potential molecular regulatory mechanisms of physical exercise in improving neurological function after cerebral ischemia in rats, but whether the timing of exercise can also impact activation of the ERK signaling pathway remains to be elucidated in future experiments.

c-Fos is a proto-oncogene, and the rapid and transient expression of the c-Fos protein can be induced by ischemia, hypoxia, injury and drug action. c-Fos is a transcription factor that regulates the expression of downstream target genes. It is involved in an array of important biological processes, including cell proliferation, differentiation, survival and regulation of axon elongation $(40,41)$. Studies have demonstrated that low frequency electrical stimulation can induce the expression of c-Fos to improve neurological function (42). In addition, it has been reported that high levels of c-Fos serve an important role in the differentiation and maturation of cerebellar granule neurons (43). c-Fos can also promote the expression of NGF to enhance neuronal repair (44), accelerate the reconstruction of neural networks and promote neuritis (45). Certain studies have identified that physical exercise promotes the expression of c-Fos in the hippocampus of rats, and may be associated with age and exercise intensity $(46,47)$. However, c-Fos has been linked to apoptosis (48); therefore, there has been some controversy over whether c-Fos is protective or damaging in cerebral ischemia. The results of the present study revealed that physical exercise increased the expression of c-Fos and improved neurological function in rats, and that treatment with U0126 inhibited c-Fos expression. These results indicated that c-Fos may be involved in regulating the proliferation and differentiation of NSCs, and that physical exercise can upregulate the expression of c-Fos to protect brain cells.

There are some limitations to the present study. Firstly, the experiments only utilized one exercise regime, and whether or not exercise type, intensity, duration and time of intervention could have different effects on activation of the ERK signaling pathway remains to be determined. Secondly, the experiments revealed that U0126 did not completely inhibit proliferation and differentiation of endogenous NSCs, suggesting that other 
signaling pathways may be involved. These questions will be explored in future experiments.

In conclusion, the present study demonstrated that physical exercise increased the number of $\mathrm{BrdU}^{+} / \mathrm{NeuN}^{+}$and $\mathrm{BrdU}^{+} / \mathrm{GFAP}^{+}$cells in the hippocampus of rats following cerebral infarction. Physical exercise promoted proliferation and differentiation of endogenous NSCs via the ERK signaling pathway by upregulating the expression of CDK4, Cyclin $\mathrm{D} 1$ and $\mathrm{p}-\mathrm{Rb}$, but downregulating the expression of $\mathrm{P}-16$, to improve neurological function. These results will aid further understanding of the mechanisms underlying the beneficial neurological effects of exercise and may provide a theoretical basis for clinical rehabilitation.

\section{Acknowledgements}

The authors would like to thank Professor Wei Hao from the Central Laboratory at Zhujiang Hospital, Southern Medical University (Guangzhou, China) for the generous help.

\section{Funding}

No funding was received.

\section{Availability of data and materials}

The datasets used or analyzed during the current study are available from the corresponding author on reasonable request.

\section{Authors' contributions}

WL, GL and WW conceived and designed the experiments; WL, GL, JC and YZ performed the experiments; WL, JC and YS analyzed the data; WL and WW analyzed and interpreted the data, performed statistical analysis, and drafted the manuscript. All authors reviewed and approved the final manuscript.

\section{Ethics approval and consent to participate}

Procedures involving animals and their care were conducted inconformity with NIH guidelines (NIH Pub. No. 85-23, revised 1996) and was approved by Animal Care and Use Committee of the Southern Medical University (Guangzhou, China).

\section{Patient consent for publication}

Not applicable.

\section{Competing interests}

The authors declare that they have no competing interests.

\section{References}

1. Shinozuka K, Dailey T, Tajiri N, Ishikawa H, Kim DW, Pabon M, Acosta S, Kaneko Y and Borlongan CV: Stem cells for neurovascular repair in stroke. J Stem Cell Res Ther 4: 12912, 2013.

2. Ambroginia P, Lattanzi D, Ciuffoli S, Betti M, Fanelli M and Cuppini R: Exercise and environment exploration affect synaptogenesis in adult-generated neurons in the rat dentate gyrus: Possible role of BDNF. Brain Res 1534: 1-12, 2013.
3. Tsai MJ, Tsai SK, Huang MC, Liou DY, Huang SL, Hsieh WH, Huang WC, Huang SS and Cheng H: Acidic FGF promotes neurite outgrowth of cortical neurons and improves neuroprotective effect in a cerebral ischemic rat model. Neuroscience 305: 238-247, 2015.

4. Gage FH: Mammalian neural stem cells. Science 287: 1433-1438, 2000.

5. Yang YR, Chang HC, Wang PS and Wang RY: Motor performance improved by exercises in cerebral ischemic rats. J Mot Behav 44: 97-103, 2012

6. Matsuda F, Sakakima H and Yoshida Y: The effects of early exercise on brain damage and recovery after focal cerebral infarction in rats. Acta Physiol (Oxf) 201: 275-287, 2011.

7. Luo J, Hu X, Zhang L, Li L, Zheng H, Li M and Zhang Q: Physical exercise regulates neural stem cells proliferation and migration via SDF-1 $\alpha /$ CXCR4 pathway in rats after ischemic stroke. Neurosci Lett 578: 203-208, 2014.

8. Tang Q, Ye T, Zhu LW, Wu XJ, Li HY and Tian Y: Effects of acupuncture-rehabilitation therapy on neurological function and extracellular signal-regulated $\mathrm{Ki}$-nase $1 / 2$ signaling pathway after focal cerebral ischemia in rats. Chin J Rehabil Theory Pract 23: 27-31, 2017.

9. Weeber EJ and Swear JD: Molecular neurobiology of human cognition. Neuron 33: 845-848, 2002.

10. Kilic U, Yilmaz B, Reiter RJ, Yüksel A and Kilic E: Effects of memantine and melatonin on signal transduction pathways vascular leakage and brain injury after focal cerebral ischemia in mice. Neuroscience 237: 268-276, 2013.

11. Li DJ, Li YH, Yuan HB, Qu LF and Wang P: The novel exercise-induced hormone irisin protects against neuronal injury via activation of the Akt and ERK1/2 signaling pathways and contributes to the neuroprotection of physical exercise in cerebral ischemia. Metabolism 68: 31-42, 2017.

12. Longa EZ, Weinstein PR, Carlson S and Cummins R: Reversible middle cerebral artery occlusion without craniotomy in rat. Stroke 20: 84-91, 1989.

13. Qin J, Gong G, Sun S, Qi J, Zhang H, Wang Y, Wang N, Wang QM, Ji Y, Gao Y, et al: Functional recovery after transplantation of induced pluripotent stem cells in a rat hemorrhagic stroke model. Neurosci Lett 554: 70-75, 2013.

14. Dragert K and Zehr EP: High-intensity unilateral dorsiflexor resistance training results in bilateral neuromuscular plasticity after stroke. Exp Brain Res 225: 93-104, 2013.

15. Shimodozono M, Noma T, Nomoto Y, Hisamatsu N, Kamada K, Miyata R, Matsumoto S, Ogata A, Etoh S, Basford JR and Kawahira K: Benefits of a repetitive facilitative exercise program for the upper paretic extremity after subacute stroke: A randomized controlled trial. Neurorehabil Neural Repair 27: 296-305, 2013.

16. Stoller O, de Bruin ED, Knols RH and Hunt KJ: Effects of cardiovascular exercise early after stroke: Systematic review and meta-analysis. BMC Neurol 12: 45, 2012.

17. Kee N, Sivalingam S, Boonstra R and Wojtowicz JM: The utility of Ki-67 and BrdU as proliferative markers of adult neurogenesis. J Neurosci Methods 115: 97-105, 2002.

18. Zhang R, Zhang Z, Wang L, Wang Y, Gousev A, Zhang L, Ho KL, Morshead C and Chopp M: Activated neural stem cells contribute to stroke-induced neurogenesis and neuroblast migration toward the infarct boundary in adult rats. J Cereb Blood Flow Metab 24: 441-448, 2004.

19. Sato K, Iwai M, Nagano I, Shoji M and Abe K: Temporal and spacial changes of BrdU immunoreactivity in amygdala kindling development. Neurol Res 24: 593-596, 2002.

20. Zhuang M,Luo J, Bai Y and Liu MF: Neural stem cell proliferation and differentiation in a neonatal rat model of hypoxia/ischemia injury Acupuncture at Ren, Du and urinary bladder meridians. Neural Regenerat Res 5: 267-272, 2010.

21. Bani-Yaghoub M, Underhill TM and Naus CC: Gap junction blockage interferes with neuronal and astroglial differentiation of mouse P19 embryonl carcinoma cells. Dev Genet 24: 69-81, 1999.

22. Chen Y and Swanson RA: Astrocytes and brain injury. J Cereb Blood Flow Metab 23: 137-149, 2003.

23. Hayakawa K, Esposito E, Wang X, Terasaki Y, Liu Y, Xing C, Ji X and Lo EH: Transfer of mitochondria from astrocytes to neurons after stroke. Nature 535: 551-555, 2016.

24. Macaluso F and Myburgh KH: Current evidence that exercise can increase the number of adult stem cells. J Muscle Res Cell Motil 33: 187-198, 2012.

25. Niwa A, Nishibori M, Hamasaki S, Kobori T, Liu K, Wake H, Mori S, Yoshino T and Takahashi H: Voluntary exercise induces neurogenesis in the hypothalamus and ependymal lining of the third ventricle. Brain Struct Funct 221: 1653-1666, 2016. 
26. DiFeo G and Shors TJ: Mental and physical skill training increases neurogenesis via cell survival in the adolescent hippocampus. Brain Res 1654: 95-101, 2017.

27. Pang Q, Zhang H, Chen Z, Wu Y, Bai M, Liu Y, Zhao Y, Tu F, Liu $C$ and Chen X: Role of caveolin-1/vascular endothelial growth factor pathway in basic fibroblast growth factor-induced angiogenesis and neurogenesis after treadmill training following focal cerebral ischemia in rats. Brain Res 1663: 9-19, 2017.

28. Ferreira AF, Real CC, Rodrigues AC, Alves AS and Britto LR: Short-term, moderate exercise is capable of inducing structural, BDNF-independent hippocampal plasticity. Brain Res 1425: 111-122, 2011.

29. Brandt MD, Maass A, Kempermann G and Storch A: Physical exercise increases Notch activity, proliferation and cell cycle exit of type-3 progenitor cells in adult hippocampal neurogenesis. Eur J Neurosci 32: 1256-1264, 2010

30. Shi HS, Xu JL, Lin GY and Wu W: Effect of $11 \mathrm{mHz}$ ultra-low frequency transcraniai magnetic stimulation on expressions of nestin and Brdu in the hippocampus of rats with focal cerebral ischemia and reperfusion. Chin J Neuromed 13: 1117-1122, 2014.

31. Kim MS, Kim KH, Lee EH, Lee YM, Lee SH, Kim HD and Kim YZ: Results of immunohistochemical staining for cell cycle regulators predict the recurrence of atypical meningiomas. J Neurosurg 121: 1189-1200, 2014.

32. Lim S and Kaldis P: Loss of Cdk2 and Cdk4 induces a switch from proliferation to differentiation in neural stem cells. Stem Cells 30: 1509-1520, 2012.

33. Brunet A, Roux D, Lenormand P, Dowd S, Keyse S and Pouysségur J: Nuclear translocation of p42/p44 mitogen-activated protein kinase is required for growth factor-induced gene expression and cell cycle entry. EMBO J 18: 664-674, 1999.

34. Avruch J: MAP kinase pathways: The first twenty years. Biochim Biophys Acta 1773: 1150-1160, 2007.

35. Chang L and Karin M: Mammalian MAP kinase signaling cascades. Nature 410: 37-40, 2001

36. Schrader LA, Birnbaum SG, Nadin BM, Ren Y, Bui D, Anderson AE and Sweatt JD: ERK/MAPK regulates the Kv4.2 potassium channel by direct phosphorylation of the pore forming subunit. Am J Physiol Cell Physiol 290: C852-C861, 2006.

37. Poddar R and Paul S: Homocysteine-NMDA receptor-mediated activation of extracellular signal-regulated kinase leads to neuronal cell death. J Neurochem 110: 1095-1106, 2009.

38. Oh MJ, Seo TB, Kwon KB, Yoon SJ, Elzi DJ, Kim BG and Namgung U: Axonal outgrowth and Erk1/2 activation by training after spinal cord injury in rats. J Neurotrauma 26: 2071-2082, 2009.
39. Tang, Qiang YE Tao, ZHU Lu-Wen, WU Xiao-Jun, LI Hong-Yu and Tian Yuan: Effects of acupuncture-rehabilitation therapy on neurological function and extracellular signal-regulated kinase $1 / 2$ signaling pathway after focal cerebral ischemia in rats. Chin J Rehabil Theory Pract 23: 27-31, 2017.

40. Güller M, Toualbi-Abed K, Legrand A, Michel L, Mauviel A, Bernuau D and Daniel F: c-Fos overexpression increases the proliferation of human hepatocytes by stabilizing nuclear Cyclin D1. World J Gastroenterol 14: 6339-6346, 2008.

41. Eriksson M and Leppä S: Mitogen-activated protein kinases and activator protein 1 are required for proliferation and cardiomyocyte differentiation of P19 embryonal carcinoma cells. J Biol Chem 277: 15992-16001, 2002.

42. Tian JB and Bishop GA: Stimulus-dependent activation of c-Fos in neurons and glia in the rat cerebellum. J Chem Neuroanat 23: $157-170,2002$

43. Eriksson M, Taskinen M and Leppä S: Mitogen activated protein kinase-dependent activation of c-Jun and c-Fos is required for neuronal differentiation but not for growth and stress response in PC I2 cells. J Cell Physiol 210: 538-548, 2007.

44. AubertN,FalluelMorel A,Vaudry D,XifroX,Rodriguez-AlvarezJ, Fisch C, de Jouffrey S, Lebigot JF, Fournier A, Vaudry H and Gonzalez BJ: PACAP and C2-cerarnide generate different AP-1 complexes through a MAP-kinase-dependent pathway: Involvement of $\mathrm{c}-\mathrm{Fos}$ in PACAP-induced $\mathrm{Bcl}-2$ expression. J Neurochem 99: 1237-1250, 2006

45. Willson ML, McElnea C, Mariani J, Lohof AM and Sherrard RM: BDNF increases homotypic olivocerebellar reinnervation and associated fine motor and cognitive skill. Brain 131: 1099-1112, 2008.

46. Kim SH, Kim H, Kim SS, Shin MS, Chang HK, Lee TH, Jang MH, Shin MC, Lee HH, Kim YP and Kim CJ: The influence of age on the treadmill exercise-induced c-Fos expression in the hippocampus of rats. Neurosci Res Communicat 35: 41-50, 2004.

47. Lee TH, Jang MH, Shin MC, Lim BV, Kim YP, Kim H, Choi HH, Lee KS, Kim EH and Kim CJ: Dependence of rat hippocampal c-Fos expression on intensity and duration of exercise. Life Sci 72: 1421-1436, 2003.

48. Vyas S, Biguet NF, Michel PP, Monaco L, Foulkes NS, Evan GI, Sassone-Corsi P and Agid Y: Molecular mechanisms of neuronal cell death: Implications for nuclear factors responding to cAMP and phorbol esters. Mol Cell Neurosci 21: 1-14, 2002.

c) (7) This work is licensed under a Creative Commons Attribution 4.0 International (CC BY 4.0) License. 